SCADide

Infer, Interpret \& Inspire Science
International Journal of Dentistry and Oral Science (IJDOS)

ISSN: $2377-8075$

\title{
Comparative Evaluation Of Enamel Re-Mineralization Potential Of An Indigenously Prepared Den- tifrice Containing 5\% Micro-Hydroxyapatite With Commercially Available Fluoridated Dentifrice By Surface Microhardness Analysis And Environmental Scanning Electron Microscopy Examination
}

Research Article

Sharath Shetty, Santosh Martande*, Dharmarajan Gopalakrishnan

Department of Periodontology, Dr. D Y Patil Vidyapeeth, Pimpri, Pune, India.

Abstract

\begin{abstract}
Objective: The purpose of this in-vitro prospective study is to assess and compare the enamel remineralization potential of indigenously prepared 5\% Micro-Hydroxyapatite (M-Hap) dentifrice with commercially available fluoridated dentifrice.

Material and Methods: Thirty Permanent single rooted extracted teeth were cleaned, polished and sectioned to remove root portion and laterembedded in acrylic blocks. All samples underwent demineralization process and later were divided into two groups for remineralization process. Group A included 15 samples treated with Test dentifrice and Group B included 15 samples treated with control dentifrice. Surface microhardness (SMH) of all the 30 samples was measuredusing Vickers Microhardness Tester at Baseline, post demineralization and post remineralization at 7 and 14 days.E-SEM analysis were performed for 5 randomly selected samples at baseline, post demineralization and post remineralization after 14 days.

Results: Intragroup comparison for both test and control group showed statistically significant differenceat different time intervals that is from baseline to demineralization followed by remineralization $(\mathrm{p}<0.01)$. Intergroup comparison did not show statistically significant difference at baseline $(p=0.646)$, post demineralization $(p=0.818)$ and post remineralization at 7 days $(p=0.429) \& 14$ days $(p=0.497)$. The remineralization evaluated by SMH and e-SEM at 7 and 14 days was marginally better in test group as compared to control group.

Conclusion: Indigenously prepared dentifrice containing 5\% Micro-hydroxyapatite showed marginally better potential for enamel remineralization as compared to commercially available Fluoridated dentifrice.
\end{abstract}

Keywords: Enamel Remineralization; Micro-Hydroxyapatite Dentifrice; Fluoride Dentifrice; Scanning Electron Microscopy.

\section{Introduction}

Enamel is a complex mineralized tissue which is responsible to maintain the integrity of tooth. Consuming acidic foods and drinks cause enamel wear and are responsible for degradation and demineralization of enamel. Demineralization makes the tooth enamel weak \& porous and it leads to the onset of dental caries. Remineralization is the process whereby mineral enters the porous enamel structure \& makes it stronger \& denser. The remineralized material hardens into enamel. The extent to which the oral fluids are saturated with the apatite minerals dictates the dynamic process of demineralization and remineralization. Calcium $(\mathrm{Ca})$ and Phosphorous $(\mathrm{P})$ are the two main components of the mineral content of the tooth [1]. The various processes that ensure the supply of these two minerals in the remineralization process have been studied and applied to develop newer strategies of remineralizing agents that emphasize on prevention of caries. Provided ecology of the tissue is conducive; re-mineralization will become the predominant process and the lesion will head towards repair [2]. Early demineralized lesions have the potential to remineralise through non-invasive procedures and this can be a great advancement in clinical management of caries.

A variety of re-mineralizing agents like fluorides, casein calcium phosphopeptides, bioactive glass etc, are dispensed via variety of vehicles like Restorative Material, Pit and Fissure Sealants, Denti-

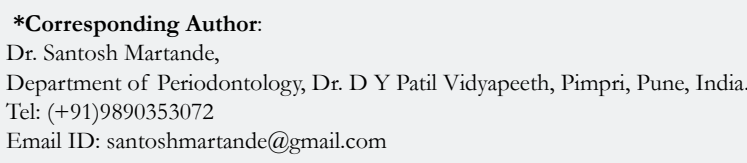

Citation: Sharath Shetty, Santosh Martande, Dharmarajan Gopalakrishnan. Comparative Evaluation Of Enamel Re-Mineralization Potential Of An Indigenously Prepared Dentifrice Containing 5\% Micro-Hydroxyapatite With Commercially Available Fluoridated Dentifrice By Surface Microhardness Analysis And Environmental Scanning Electron Microscopy Examination. Int J Dentistry Oral Sci. 2021;08(5):2446-2453. doi: http://dx.doi.org/10.19070/2377-8075-21000481

Copyright: Santosh Martande ${ }^{\circ} 2021$. This is an open-access article distributed under the terms of the Creative Commons Attribution License, which permits unrestricted use, distribution and reproduction in any medium, provided the original author and source are credited. 
frices, Chewing Gums and Mouth Rinses. Most common way of dispensing is via incorporation into dentifrices [3].

Fluoride (F) has been one of the most efficient re-mineralizing agents. Nevertheless, the over the counter use of fluoride products and widely prescribed fluorides have raised certain concerns regarding the total fluoride intake which has increased to harmfullevels. This has led to increase in incidence of dental fluorosis, noticeably in non-fluoridated areas and to a lesser extent in optimally fluoridated areas [3]. Also re-mineralization with fluoride has found to be via formation of Fluorapatite crystals and not by Hydroxyapatite crystals [4]. Hence it is essential to seek alternative and effective non-fluoride agents which can ensure an effective treatment for demineralization.

Recently, fluoride alternatives such as nano-hydroxyapatite (nanoHAp)and casein phosphopeptide (CPP) have been in limelight for their re-mineralizing properties. $\mathrm{CPP}$ with its $\mathrm{Ca}$ and $\mathrm{P}$, has high affinity for the HA of enamel, thereby enhancing its re-mineralization and is also safe to use clinically [5]. In the recent past, nanoHap has gained popularity due to its biocompatible and bioactive properties in medicine and dentistry. nano-HApbeing chemically and structurally similar to enamel minerals, their application for biomimetic repair of the demineralized enamel directly has received utmost importance $[6,7]$. In the 1980 s toothpastes containing nano-Hap were first introduced and tested in Japan (e.g. Apadent, Apagard, and others by Sangi Co., Ltd., Tokyo).

Till date there is no indigenously prepared dentifrice containing Nano - Hap or Micro-Hap available in India. The major limitation of using Nano-Hap is the higher cost factor in Indian scenario.

Hence the purpose of this in-vitro prospective study is to assess and compare the enamel remineralization potential of indigenously prepared 5\% Micro-HAp dentifrice with commercially available fluoridated dentifrice.

\section{Materials And Methods}

The studywas a Comparative In-vitro Prospective study conducted over a period of 3 months at Department of Periodontology, Dr. D Y Patil Vidyapeeth, Pune. Thirty Permanent single rooted extracted teeth were collected from the Department of Oral and Maxillofacial Surgery, Dr. D Y Patil Vidyapeeth, Pune.

\section{Inclusion Criteria:}

- Premolar teeth indicated for extractions (Orthodontic reasons) - The crown portion of the tooth should be sound and intact with normal anatomical features.

\section{Exclusion Criteria:}

- Teeth having developmental defects.

- Visible and detectable carious lesions on teeth

- White spot lesions on teeth

- Fractured Teeth

- Restored Teeth

- Root Canal Treated tooth

- Teeth with abrasion and erosion.

\section{Method Of Sample Allocation}

Thirty extracted teeth were allocated into 2 groups by random allocation sequence (Computer generated sequence) -

Test group: Dentifrice containing 5\% Micro- hydroxyapatite. (Dentifrice A)

Control Group: Commercially available Fluoridated Dentifrice. (Dentifrice C)

The dentifrices were dispensed in coded sterile Eppendorf tubes (Dentifrice A \& C) and provided to the blinded investigator. The investigator performing the procedure, Outcome assessor and Statistician will be blinded to the products used for remineralization.

\section{Places where the study procedures were conducted:}

Sample storage, Sample preparation, Demineralization process, Re-mineralization process -Department of Periodontology in Dr. D.Y. Patil Vidyapeeth.

Demineralization \& remineralization solution preparation: Department of Oral Pathology, Dr. D.Y. Patil Dental College, Pimpri.

Vicker's Microhardness Testing machine (VMT) - Praj Metallurgy Lab, Pune.

E-SEM- ICON Analytical, Mumbai.

\section{Procedure}

\section{Sample Collection \& Specimen Preparation}

- The freshly extracted teeth first were cleaned to remove any adherent material like plaque, calculus, granulation tissue.

- Then with a slow speed diamond bur, teeth were sectioned $1 \mathrm{~mm}$ below the CEJ

- Sectioned roots were discarded and crowns were preserved in $10 \%$ formalin until further use.

- The buccal surface was polished using polishing paste and rubber cup.

- Customized cylindrical plastic moulds were made and self-cure acrylic resin was poured in them. Crowns were embedded in the resin such that the buccal surfaces faced upwards and parallel to the horizontal plane.Following the placement a $5 \mathrm{~mm} \times 5 \mathrm{~mm}$ window of exposing the enamel surface was created in the middle of the sample.

- Baseline surface microhardness (B-SMH) of all the 30 samples were measured by using Vickers Microhardness Tester [8].

\section{Baseline Surface Microhardness (B-SMH) Measurement.} [8]

- B-SMH were checked with Vicker's Microhardness Tester (Micro Vicker's hardness Tester, Reichert, Austria) for all the tooth samples in the area of the working window.

- For the quantitative assessment, the indentations were made with VMT at the rate of $100 \mathrm{gf}$ (gram force) load with a holding period 
of 15 seconds. The average microhardness of the specimen will be determined from three indentations to avoid any discrepancy. - The minimum distance of 150 micrometer was ensured between adjacent indentations in order to avoid measurement errors.

\section{Baseline E-SEM Analysis [10]}

- 5 Samples each from Test and control group were randomly selected after VMT for SEM analysis and marked with acrylic paints of two different colours (for Test/Control) for ease of identification during subsequent analysis. So that these 5 samples were constant throughout the entire study period for both the groups.

- The E-SEM (Carl Zeiss, Oxford Instrument,INCA - Oxford Software Package) was used and images were obtained at 500x magnification 1.

\section{Preparation of Demineralising Solution [9]}

- The composition of demineralizing solution used was as follows:

\section{Demineralizing solution}

- $2.2 \mathrm{mM}$ calcium chloride $\left(\mathrm{CaCl}_{2} .2 \mathrm{H}_{2} \mathrm{O}\right)$

- $2.2 \mathrm{mM}$ monosodium phosphate $\left(\mathrm{NaH}_{2} \mathrm{PO}_{4} .7 \mathrm{H}_{2} \mathrm{O}\right)$

- $0.05 \mathrm{M}$ lactic acid

The final $\mathrm{pH}$ was adjusted to 4.5 with $50 \%$ sodium hydroxide $(\mathrm{NaOH})$.

Artificial saliva (Wet Mouth, ICPA) with the following composition was procured.

- Sodium carboxymethyl cellulose- $0.5 \% \mathrm{w} / \mathrm{v}$

- Glycerin- 30\% w/v

\section{Lesion Formation (Demineralising Process) [9]}

- All the samples (of both the groups) were individually immersed in the demineralising solution $(20 \mathrm{ml})$ for $15 \mathrm{~min}$ at $370 \mathrm{C}$ using an incubator. This demineralising process was intended to produce subsurface lesions in the enamel.

- After 48 hours of incubation in the demineralizing solution, the teeth were washed with deionized water, dried with the help of an air syringe, and placed in different clean glass containers.

- After Demineralisation for 48 hours, VMT was used to assess Surface Microhardness values (D-SMH), and then followed by E-SEM analysis of the previously selected 5 samples from each group.

\section{Remineralization Process [9]}

\section{Remineralization Process (for Group A and Group B):}

- All the 30 samples (both of Group A and Group B) were subjected to remineralization process as below:

\section{Group A:}

In the Group A group, paste A was applied by applicator tips on all the 15 samples every 24 hours for 14 days and left on for
4 minutes following which the samples were thoroughly washed with deionized water and placed in artificial saliva.

$>$ Just prior to immersion of samples every 24 hours artificial saliva was changed with fresh solution.

\section{Group B:}

In the Group B group, paste C was applied by applicator tips on all the 15 samples every 24 hours for 14 days and left on for 4 minutes following which the samples were thoroughly washed with deionized water and placed in artificial saliva

$>$ Artificial saliva was changed every 24 hours just before immersion of freshly treated samples.

- After 14 cycles of remineralization for both the groups, the surface microhardness was assessed using VMT (R-SMH).

- This was then followed by E-SEM of the previously selected 10 samples (5 samples of Group A and 5 samples of Group B) to assess the surface changes.

\section{Method Of Data Analysis}

- The data obtained was subjected to statistical analysis using the IBM Statistical Program for Social Sciences Version 18.0 (SPSS Inc., Chicago Illinois, USA).

- The results related to comparison between the toothpastes were analysed by unpaired t-test (normality of data to be checked).

- Comparison between baseline, post-demineralisation and postremineralisation results were analysed by two way ANOVA and post hoc test (normality of data to be checked).

- For the entire evaluation, $\mathrm{p}<0.05$ was considered as statistically significant.

\section{Results}

\section{VHN Analysis:}

\section{Dentifrice A:}

Mean baseline VHN was $337.73 \pm 16.166$

Mean Demineralisation VHN was $219.66 \pm 29.244$

Mean remineralisation VHN after 7 days was $282.53 \pm 47.062$

Mean remineralisation VHN after 14 days was $307.73 \pm 33.703$

Mean remineralisation value of paste A after 7 days was 289.53 which was statistically significant compared to mean demineralisation value.

Mean remineralisation value of paste A after 14 days was 307.73 which was statistically significant compared to mean demineralisation value.

But the comparison of remineralisation values of paste $A$ at 7 and 14 days were compared by ANOVA and post hoc test and result was not statistically significant.

\section{Dentifrice C:}

Mean baseline VHN was $340.27 \pm 13.646$

Mean Demineralisation VHN was $217 \pm 13.646$

Mean remineralisation VHN after 7 days was $276 \pm 45.264$ 
Table 1. Distribution and comparison of study samples in two different commercially available products.

\begin{tabular}{|c|c|c|c|c|c|c|c|}
\hline \multicolumn{8}{|c|}{ Unpaired t test } \\
\hline \multicolumn{2}{|c|}{ Sample } & N & Mean & Std. Deviation & t & Mean diff & P value \\
\hline \multirow{2}{*}{\begin{tabular}{c} 
baseline \\
\cline { 2 - 9 }
\end{tabular}} & $\mathrm{C}$ & 15 & 340.27 & 13.646 & 0.464 & 2.533 & 0.646 \\
\hline \multirow{2}{*}{$\begin{array}{c}\text { deminerali- } \\
\text { zation }\end{array}$} & $\mathrm{C}$ & 15 & 337.4 & 16.166 & & & \\
\cline { 2 - 9 } & $\mathrm{A}$ & 15 & 219.67 & 29.244 & & & 0.818 \\
\hline $\begin{array}{c}\text { Reminerali- } \\
\text { sation 7th }\end{array}$ & $\mathrm{C}$ & 15 & 276 & 45.264 & -0.803 & -13.533 & 0.429 \\
\cline { 2 - 9 } & $\mathrm{A}$ & 15 & 282.53 & 47.062 & & & \\
\hline $\begin{array}{c}\text { Reminerali- } \\
\text { sation 14th }\end{array}$ & $\mathrm{C}$ & 15 & 299.13 & 34.707 & -0.688 & -8.6 & 0.497 \\
\cline { 2 - 9 } & $\mathrm{A}$ & 15 & 307.73 & 33.703 & & & \\
\hline
\end{tabular}

Table 2. Distribution of study samples in group $\mathrm{C}$ products at different time interval usingrepeated measures ANOVA test.

\begin{tabular}{|c|c|c|c|c|}
\hline & Mean & Std. Deviation & F & P value \\
\hline baseline & 340.27 & 13.646 & & \\
\cline { 1 - 3 } demineralization & 217 & 33.535 & \multirow{6}{*}{68.864} & $<0.001$ \\
\cline { 1 - 3 } rem7th & 276 & 45.264 & & \\
\cline { 1 - 3 } rem14th & 299.13 & 34.707 & & \\
\hline
\end{tabular}

Table 3. Distribution and comparison of study samples in group C products at different time interval using post hoc LSD test.

\begin{tabular}{|c|c|c|c|}
\hline \multirow{4}{*}{$(\mathbf{I}) \mathbf{f}$} & $\mathbf{( J )} \mathbf{f}$ & Mean Difference (I-J) & P value \\
\hline \multirow{4}{*}{ Baseline } & Demineralisation & $123.267^{*}$ & $<.001$ \\
\cline { 2 - 4 } & Remineralisation 7th day & $64.267^{*}$ & $<.001$ \\
\cline { 2 - 4 } & Remineralization 14th day & $41.133^{*}$ & $<.001$ \\
\hline \multirow{4}{*}{\begin{tabular}{c} 
Bemineralisation \\
\cline { 2 - 4 }
\end{tabular}} & Baseline & $-123.267^{*}$ & $<.001$ \\
\cline { 2 - 4 } & Remineralisation 7th day & $-59.000^{*}$ & $<.001$ \\
\hline \multirow{3}{*}{$\begin{array}{c}\text { Remineralisation } \\
\text { 7th day }\end{array}$} & Baseline & $-82.133^{*}$ & $<.001$ \\
\cline { 2 - 4 } & Demineralisation & $-64.267^{*}$ & $<.001$ \\
\cline { 2 - 4 } & Remineralization 14th day & $-23.133^{*}$ & $<.000^{*}$ \\
\hline \multirow{3}{*}{$\begin{array}{c}\text { Remineralization } \\
\text { 14th day }\end{array}$} & Baseline & $-41.133^{*}$ & $<.003$ \\
\cline { 2 - 4 } & Demineralisation & $82.133^{*}$ & $<.001$ \\
\cline { 2 - 4 } & Remineralisation 7th day & $23.133^{*}$ & 0.003 \\
\hline
\end{tabular}

Table 4. Distribution of study samples in group A products at different time interval usingrepeated measures ANOVA test.

\begin{tabular}{|c|c|c|c|c|}
\hline \multicolumn{4}{|c|}{ Descriptive Statistics } & \\
\hline & Mean & Std. Deviation & F & P value \\
\hline Baseline & 337.73 & 16.166 & & \\
\cline { 1 - 3 } Demineralization & 219.66 & 29.244 & \multirow{6}{*}{64.838} & \multirow{2}{*}{$<.001$} \\
\cline { 1 - 3 } rem7th & 289.53 & 47.062 & & \\
\hline rem14th & 307.73 & 33.703 & & \\
\hline
\end{tabular}

Mean remineralisation VHN after 14 days was $299.13 \pm 34.707$

Mean remineralisation value of paste $C$ after 7 days was 276 which was statistically significant compared to mean demineralisation value.

Mean remineralisation value of paste $C$ after 14 days was 299.13 which was statistically significant compared to mean demineralisation value.

But the comparison of remineralisation values of Paste $\mathrm{C}$ at 7 and 14 days were compared by ANOVA and post hoc test and result was not statistically significant. 
Table 5. Distribution and comparison of study samples in group A products at different time interval using post hoc LSD test.

\begin{tabular}{|c|c|c|c|}
\hline (I) factor1 & (J) factor1 & Mean Difference (I-J) & P value \\
\hline \multirow{4}{*}{ Baseline } & Demineralisation & $118.067^{*}$ & $<.001$ \\
\cline { 2 - 4 } & Remineralisation 7th day & $48.200^{*}$ & $<.001$ \\
\cline { 2 - 4 } & Remineralization 14th day & $30.000^{*}$ & $<.001$ \\
\hline \multirow{4}{*}{ Demineralisation } & Baseline & $-118.067^{*}$ & $<.001$ \\
\cline { 2 - 4 } & Remineralisation 7th day & $-69.867^{*}$ & $<.001$ \\
\cline { 2 - 4 } & Remineralization 14th day & $-88.067^{*}$ & $<.001$ \\
\hline \multirow{3}{*}{ Remineralisation } & Baseline & $-48.200^{*}$ & $<.001$ \\
\cline { 2 - 4 } 7th day & Demineralisation & $69.867^{*}$ & $<.001$ \\
\cline { 2 - 4 } & Remineralization 14th day & $-18.200^{*}$ & $<.001$ \\
\hline \multirow{3}{*}{$\begin{array}{c}\text { Remineralization } \\
\text { 14th day }\end{array}$} & Baseline & $-30.000^{*}$ & $<.001$ \\
\cline { 2 - 4 } & Demineralisation & $88.067^{*}$ & $<.001$ \\
\cline { 2 - 4 } & Remineralisation 7th day & $18.200^{*}$ & $<.001$ \\
\hline
\end{tabular}

\section{Inter group comparison:}

The mean baseline values were compared between dentifrice $\mathrm{A}$ and Dentifrice C. Mean demineralisation values between Dentifrice $A$ and Dentifrice $C$ was not statistically significant $(\mathrm{p}=$ 0.646).

The mean demineralisation value of Dentifrice A is 219.66 and Dentifrice $C$ is 217 . There was no statistically significant difference between mean demineralisation values for Dentifrice $A$ and Dentifrice C. $(p=0.818)$.

The remineralisation values at 7 and 14 days for Dentifrice $A$ and dentifrice $\mathrm{C}$ were compared using ANOVA and post hoc test. There was no statistically significant difference between both the dentifrices after 7 days $(p=0.429) \& 14$ days $(p=0$. of remineralisation $(\mathrm{p}=0.497)$.

\section{E-SEM Results}

Qualitative assessment of study outcome was evaluated by ESEM at 40X, 500X (3), 1000X, 2000X. All 5 samples in each group were assessed at baseline, after demineralisation, post remineralisation after 14 days. And 6 readings were taken at $40 \mathrm{X}$, 500X, 1000X, 2000X.

Environmental scanning electron microscopic picture showed surface topographical image of demineralized surface and surface after treatment with Dentifrice $C$ in Fig. 1 and Fig. 2 while Fig 3 and Fig. 4 shows surface topographical image of demineralized surface and surface after treatment with Dentifrice A. Surface topographical view of demineralized surface showed the exposed cross-sectionally cut rod ends arranged in the form of keyhole or hexagonal pattern due to removal of aprismatic surface enamel by the action of demineralizing agent. On the other hand, the surface topographical view after treatment with Dentifrice A (after remineralization) showed the loss of keyhole pattern in various degrees, which is attributed to the redisposition of the calcium and phosphate ions in the aprismatic manner. Fig. 1 showed complete loss of keyhole or hexagonal pattern of enamel rod suggesting complete re-mineralization takes place in the sample. While in Fig. 2 there is patchy loss of keyhole or hexagonal pattern. This suggests that Dentifrice A initiated moderate degree of the re-mineralization. When we compare the quality of enamel surface after the application of Dentifrice $A$ and Dentifrice $C$ under ESEM (Qualitative Assessment), loss of keyhole pattern due to re-deposition of calcium and phosphate ions in the aprismatic manner is better in Dentifrice A as compared to Dentifrice C. These findings were in accordance with the VHN values (Quantitative Assessment) even though the difference is not statistically significant

\section{Interpretation}

Distribution and inter group Comparison of study samples in two different commercially available products showed statistically insignificant $(\mathrm{P}>0.05)$ results at different time interval. However, the mean score of Dentifrice A product was slightly higher than Dentifrice C.

Distribution and intra group Comparison of study samples in two different commercially available products showed statistically significant $(\mathrm{P}<0.05)$ results at different time interval in both the group. However, the mean score of Dentifrice A product showed better score in different time interval compared to Dentifrice C.

\section{Discussion}

Early detection and prevention of tooth demineralization remains the prime focus of preventive dentistry before restorative dentistry comes into picture. Extensive research has been performed and is currently undergoing to devise ways to limit enamel demineralisation and promote enamel remineralisation thereby facilitating good dental and oral hygiene.

Clinical trials are considered the gold standard in research methodology but standardised in-vitro study models are the conventional and easier method of assessing enamel remineralisation efficacy in cariology research [11]. The current study was designed as an in-vitro study model to evaluate enamel re-mineralization potential of an indigenously prepared dentifrice containing 5\% Nano-hydroxyapatite with commercially available Fluoridated 
Paste C

Figure 1.

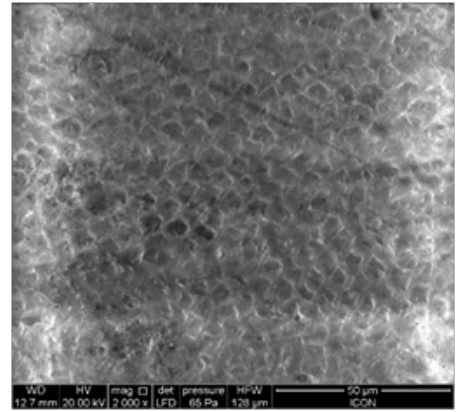

After Demineralization

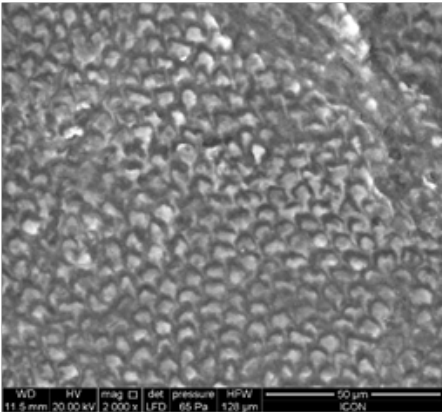

After Remineralization

Figure 2.

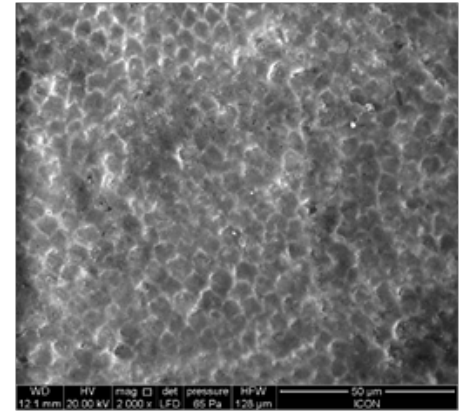

After Demineralization

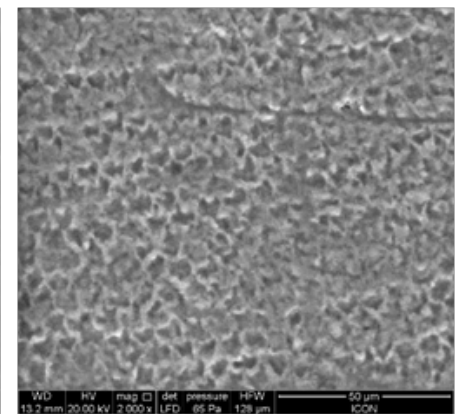

After Remineralization

\section{Dentifrice A}

Figure 3.

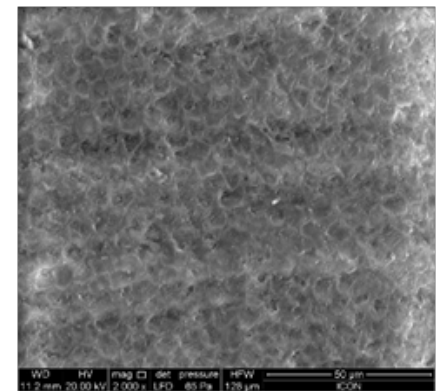

After Demineralization

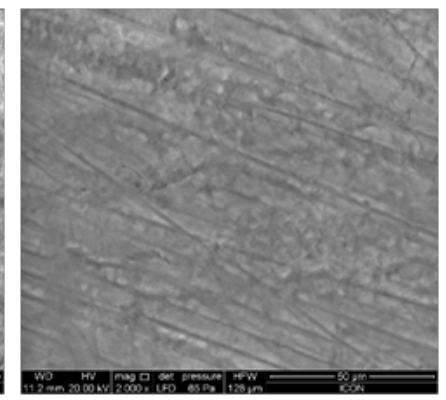

After Remineralization

Figure 4 .

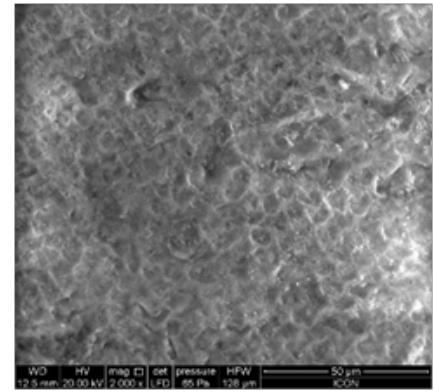

After Demineralization

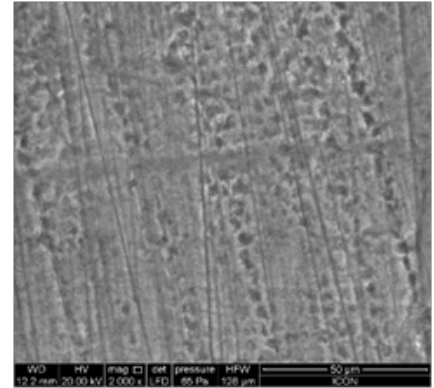

After Remineralization dentifrice.

The study protocol adopted demineralization cycle followed by remineralization cycle with individual dentifrices. The final $\mathrm{pH}$ of demineralising solution was adjusted to 4.5 with $50 \%$ sodium hydroxide $(\mathrm{NaOH})$. Each sample underwent 30 minutes of demineralization cycle. After which each sample was subjected to VHN analysis and 5 samples from each group were subjected to E-SEM analysis. After demineralization samples underwent topical application with respective dentifrice every 24 hours for 14 days and left on for 4 minutes following which the samples were thorough- ly washed with deionized water and placed in artificial saliva. After which each sample was subjected to VHN analysis after 7 and 14 days and 5 samples from each group were subjected to E-SEM analysis after 14 days. This entire protocol was in accordance with the study by Patil $\mathrm{N}$ et al. [9]

Variety of techniques have been implied for assessing enamel remineralisation. Quantitatively the mineral content and hardness profiles are assessed. Qualitatively Polarized Light Microscopy (PLM) and SEM are used for assessment [12]. 
The study used Vickers Hardness Number (VHN) for surface microhardness (SMH) evaluation. SMH evaluation is a simple, quick and easy to measure non destructive method, reflecting mineral changes occurring during the demineralization and remineralization cycles. The method also permits repeated measurements of the same specimen over a given period of time thereby reducing the experimental variation [10].

Demineralisation process was carried out immersing the samples in the demineralizing solution.

The average demineralisation VHN for M-HAp was 219.66 and average Demineralisation $\mathrm{VHN}$ for $\mathrm{NaF}$ is 217.

Following Remineralisation process all 30 samples (15 each group) were subjected to VHN.

After 7 days the average remineralisation VHN for M-HAp was 289.53 and average remineralisation $\mathrm{VHN}$ for $\mathrm{NaF}$ is 276

After 14 days the average remineralisation VHN for M-HAp was 307.73 and average remineralisation VHN for $\mathrm{NaF}$ is 299 .

To the best of our knowledge it's a first kind of study where MHAp (M-HAp) is compared with $\mathrm{NaF}$.

In this study there was a statistically significant difference between SMH in M-HAp after 14 days of remineralisation process $(\mathrm{p}=0.001)$.

In this study there was a statistically significant difference between $\mathrm{SMH}$ in $\mathrm{NaF}$ after 14 days of remineralisation process $(\mathrm{p}=0.001)$ This difference of remineralisation is statistically insignificant between M-HAp \& NaF.

Swarup J S et al [8] in 2012. conducted a study where fluoride was compared with \& N-HAp. The mean difference in SMH after 14 days of remineralisation in this study was $13.5 \pm 1.3$ (in fluoride group) and $27 \pm 2.3$ in (N-HAp). In our study Fluoride group the mean VHN difference was $82.13 \pm 1.17$ and for M-HAp group was $88.06 \pm 4.45$. These results obtained are better than the above study.

Huang et al [7] in 2009 conducted a study to compare the remineralising efficacy of varying concentrations of N-HAp $(1 \%, 5 \%, 10 \%, 15 \%)$ with $1000 \mathrm{ppm} \mathrm{NaF}$ solutions. The mean difference in SMH after 14 days of remineralisation in this study was $174.7 \pm 8.9$ (in fluoride group), $45.9 \pm 5.6$ in (1\% N-HAp), $73.8 \pm 4.8$ (5\% N-HAp), $124.6 \pm 1.9$ (10\% N-HAp) and $124.5 \pm 0.3(15 \% \mathrm{~N}-$ HAp). In our study the mean VHN difference for M-HAp group was $88.06 \pm 4.45$. These results obtained are in accordance with 5 $\%$ nano HAp group of the above study.

The above comparisons leads to a observation that remineralisation potential of $5 \% \mathrm{M}-\mathrm{HAp}$ will be similar to that of $5 \% \mathrm{~N}$ HAp, In this context, Future studies are required to evaluate different percentages of M-HAp versus N-HAp.

Qualitative assessments revealed nanocrystals of HA are adherent to the demineralization pores. A uniform apatite layer was formed on the demineralized by these adherent nanocrystals which aggregated and grew into microclusters. Prismatic and interprismatic enamel structures completely covered the enamel surfaces. (SEM N-HAp).

Even though the quantitative assessment of $5 \% \mathrm{M}-\mathrm{HAp}$ in our study is in accordance with 5\% N-HAp study by Huang et al and better than $1 \% \mathrm{~N}-\mathrm{HAp}$ study by huang et al, we failed to show the superiority of quantitative assessment of $5 \%$ M-HAp with fluoride group. This difference and may be due to no similar points taken on the specimens before and after demineralisation and remineralisation for $\mathrm{VHN}$ assessment. This is due to inherent disadvantage assosciated with this technique.

\section{Future Directions}

1. Future research is needed to compare different concentrations of M-HAp with different concentrations of N-HAp along with Quantitative assessment of comparison between different concentrations of M-HAp.

2. Longitudinal studies evaluating the remineralizing efficacy of dentifrices for 28 days and further.

\section{Conclusions}

Within the limitations of the study, based on the surface microhardness and environmental scanning electron microscope analysis it can be concluded that:

1. $\mathrm{NaF}$ dentifrice showed significant increase in surface microhardness at 7 and 14 days post remineralization cycle after undergoing demineralization.

2. M-HAp dentifrice showed significant increase in surface microhardness at 7 and 14 days post remineralization cycle after undergoing demineralization.

3. M-HAp dentifrice showed slightly better improvement in surface microhardness at 7 and 14 days post remineralization cycle after undergoing demineralization (though statistically insignificant).

4. M-HAp dentifrice is better than $\mathrm{NaF}$ dentifrice via Qualitative assessment by using ESEM (Statistical analysis not applicable).

5. Conclusions drawn from our study with literature evidence is that, remineralisation potential of $5 \%$ M-HAp (quantitative assessment) is similar to $5 \%$ nano HAp.

\section{Acknowledgements}

The Authors thank Anchor Health and Beauty Care Pvt. Ltd, Mumbai INDIA for providing funds and the dentifrice samples to carry out the study. The Authors mention their special thanks to Mr. Sanjay Shah, MD and Dr. Vivek Patwardhan, Head R\&D, Anchor Consumer Products Pvt. Ltd, Mumbai, INDIA for their constant support during the tenure of the study.

\section{References}

[1]. Nithin, MG.\& Joseph, J. Effect of nano-hydroxyapatite on remineralization of enamel-a systematic review.Int J Dent Res Develop, 2015;5(2):13-20.

[2]. Pearce EI, Moore AJ. Remineralization of softened bovine enamel following 
treatment of overlying plaque with a mineral-enriching solution. J Dent Res. 1985 Mar;64(3):416-21. Pubmed PMID: 3855891.

[3]. Pendrys DG. Dental fluorosis in perspective. J Am Dent Assoc. 1991 Sep;122(9):63-6. Pubmed PMID: 1918669.

[4]. Lata S, Varghese NO, Varughese JM. Remineralization potential of fluoride and amorphous calcium phosphate-casein phospho peptide on enamel lesions: An in vitro comparative evaluation. J Conserv Dent. 2010 Jan;13(1):42-6. Pubmed PMID: 20582219.

[5]. Zhang L, Li Z, Dong Y. Experimental study of phosphopeptide in promoting tooth remineralization. Chin J Dent Res. 2000 May;3(1):27-30. Pubmed PMID: 11314337.

[6]. Li L, Pan H, Tao J, Xu X, Mao C, Gu X, et al. Repair of enamel by using hydroxyapatite nanoparticles as the building blocks. Journal of Materials Chemistry. 2008;18(34):4079-84.

[7]. Huang SB, Gao SS, Yu HY. Effect of nano-hydroxyapatite concentration on remineralization of initial enamel lesion in vitro. Biomed Mater. 2009 Jun;4(3):034104. Pubmed PMID: 19498220.
[8]. Swarup JS, Rao A. Enamel surface remineralization: Using synthetic nanohydroxyapatite. Contemporary clinical dentistry. 2012 Oct;3(4):433.

[9]. Patil N, Choudhari S, Kulkarni S, Joshi SR. Comparative evaluation of remineralizing potential of three agents on artificially demineralized human enamel: An in vitro study. J Conserv Dent. 2013 Mar;16(2):116-20. Pubmed PMID: 23716961.

[10]. Soares R, De Ataide ID, Fernandes M, Lambor R. Assessment of enamel remineralisation after treatment with four different remineralising agents: A Scanning Electron Microscopy (SEM) Study. Journal of clinical and diagnostic research: JCDR. 2017 Apr;11(4):ZC136

[11]. Buzalaf MA, Hannas AR, Magalhães AC, Rios D, Honório HM, Delbem AC. $\mathrm{pH}$-cycling models for in vitro evaluation of the efficacy of fluoridated dentifrices for caries control: strengths and limitations. J Appl Oral Sci. 2010 Jul-Aug;18(4):316-34. Pubmed PMID: 20835565.

[12]. Montasser MA, El-Wassefy NA, Taha M. In vitro study of the potential protection of sound enamel against demineralization. Prog Orthod. 2015;16:12. Pubmed PMID: 26061985. 